\title{
Translating Evidence into Practice: ACOs' Use of Care Plans for Patients with Complex Health Needs
}

\author{
Taressa K. Fraze, $P h D^{1,2}$ (D), Laura B. Beidler, $M P H^{2}$, \\ Adam D. M. Briggs, B.M.B.Ch., D.Phil. ${ }^{2,3,4}$, and Carrie H. Colla, PhD 2
}

'Department of Family and Community Medicine, University of California, San Francisco, San Francisco, CA, USA; ${ }^{2}$ The Dartmouth Institute for Health Policy and Clinical Practice, Geisel School of Medicine, Dartmouth College, Hanover, NH, USA; ${ }^{3}$ Division of Health Sciences, Warwick Medical School, University of Warwick, Coventry, UK; ${ }^{4}$ The Health Foundation, London, UK.

BACKGROUND: Care plans are an evidence-based strategy, encouraged by the Centers for Medicare and Medicaid Services, and are used to manage the care of patients with complex health needs that have been shown to lead to lower hospital costs and improved patient outcomes. Providers participating in payment reform, such as accountable care organizations, may be more likely to adopt care plans to manage complex patients.

OBJECTIVE: To understand how Medicare accountable care organizations (ACOs) use care plans to manage patients with complex clinical needs.

DESIGN: A qualitative study using semi-structured interviews with Medicare ACOs.

PARTICIPANTS: Thirty-nine interviews were conducted across 18 Medicare ACOs with executive-level leaders and associated clinical and managerial staff.

APPROACH: Development, structure, use, and management of care plans for complex patients at Medicare ACOs.

KEY RESULTS: Most (11) of the interviewed ACOs reported using care plans to manage care of complex patients. All care plans include information about patient history, current medical needs, and future care plans. Beyond the core elements, care plans included elements based on the ACO's planned use and level of staff and patient engagement with care planning. Most care plans were developed and maintained by care management (not clinical) staff.

CONCLUSIONS: ACOs are using care plans for patients with complex needs, but their use of care plans does not always meet the best practices. In many cases, ACO usage of care plans does not align with prescribed best practices: ACOs are adapting use of care plans to better fit the needs of patients and providers.

KEY WORDS: primary care; accountable care organizations; complex patients; care plans.

Prior Presentations A portion of this manuscript was presented at the AcademyHealth Annual Research Meeting in Washington DC on June 2, 2019.

Electronic supplementary material The online version of this article (https://doi.org/10.1007/s11606-020-06122-4) contains supplementary material, which is available to authorized users.

Received June 7, 2019

Revised April 23, 2020

Accepted August 7, 2020

Published online October 1, 2020
$\mathrm{J}$ Gen Intern Med 36(1):147-53

DOI: $10.1007 / \mathrm{s} 11606-020-06122-4$

(c) Society of General Internal Medicine 2020

\section{INTRODUCTION}

Traditional physician-patient relationships included many aspects of patient care that can be lost in today's fragmented or team delivery models. Physicians that have long-term relationships with patients understand their histories, life situations, and goals. Yet, on average, Medicare patients see an increasing array of clinicians, both in number and in specialty. ${ }^{1,2}$ This is especially the case for patients with complex health needs ${ }^{3}$ - such as those with multiple chronic conditions, serious illnesses, or behavioral health needs - and these patients drive much of healthcare spending. ${ }^{4}$ Complex patients may need more intensive, frequent, coordinated, and comprehensive healthcare that is targeted to their clinical and socioeconomic concerns, and a thorough understanding of their history, needs, and goals is vital to successful care. ${ }^{5-8}$

Comprehensive patient care plans are an evidence-based tool used to clinically manage patients with complex health needs. Care plans can modestly improve patients' clinical and psychosocial markers including blood pressure control, depression symptoms, and perceived ability to self-manage health. ${ }^{9,}{ }^{10}$ A recent systematic review that examined 19 randomized control trials found that personalized care plans are likely most effective when interventions are more comprehensive, intense, and integrated into routine care. ${ }^{9}$ Across studies, the effects of care planning were modest-for example, they found moderate quality evidence for the impact of care planning on glycated hemoglobin (HbAlc) across nine studies (mean difference between intervention and control of $-0.24 \%)$. $^{9}$ In addition to improving clinical markers, care plans are used with the goal of reducing unnecessary hospital-based utilization. There is some evidence which suggests care plans may reduce subsequent inpatient stays: in a pre-post design, one study found a significant decrease in hospital stays (56\% reduction at 6 months) and 30-day readmissions (66\% reduction at 6 months), but more rigorous 
research is needed to fully understand the impact of care plans on utilization outcomes. ${ }^{11}$

With the hope of improving quality outcomes while reducing costs, the Centers for Medicare and Medicaid Services (CMS) has embraced the use of care plans as a marker of advanced primary care by requiring providers to use them when billing under Chronic Care Management $\operatorname{codes}^{12}$ as well as part of the $\mathrm{CPC}+{ }^{13}$ and the new Primary Care First models. ${ }^{14}$ Care plans are ideally developed after consultation with the patient and in collaboration with their broader care team including primary and specialist care. ${ }^{15}$ Care plans should serve as the centralized landing space for a given patient to ensure all care team members can easily access and contribute to comprehensive information about the patient including the patient's history, current clinical and non-clinical needs, and goals, ${ }^{5,15-18}$ and they should be driven by a patient's personal preferences and aligned with their clinical needs. ${ }^{5,17,19-21}$

Medicare, Medicaid, and commercial payers are using alternative payment models, such as accountable care organizations (ACOs) and medical homes, to promote accountability for patient needs across the care spectrum. Providers under value-based contracts have incentives to implement evidencebased interventions, such as patient care plans, that are not directly reimbursable but may impact overall spending and quality of care. ${ }^{22}$ While Medicare's ACO models have shown modest reductions in total costs of care, evidence suggests that savings may be more substantial for patients with complex clinical needs. ${ }^{23}$ Patients with complex health needs may benefit the most from care delivery transformations associated with payment reform; therefore, ACOs may use care plans as a way to realize savings via patients with complex needs who typically drive healthcare costs. ${ }^{24}$

Payers are also introducing chronic condition management billing codes ${ }^{12}$ special needs plans, and advanced primary care models ${ }^{13,14}$ to promote specialized care for complex patients. ${ }^{25}$ Yet, there is little understanding of how providers use care plans in routine clinical settings. ${ }^{26,27}$ Previous studies have typically assessed care plans as part of formalized, multifaceted interventions and have shown modest improvements to physical health, but little is known about how care plans are used outside of these formal programs. ${ }^{9}{ }^{10}$ Frontline providers may struggle to implement care plans using best practice$\mathrm{s}$-including collaborative development between primary care, specialist care, and patients - identified in previous research. In this study, we use qualitative interviews to describe how Medicare ACO providers, who have been successful at meeting savings and quality benchmarks, develop and implement processes around care plans for patients with complex health needs.

\section{METHODS}

We conducted 39 semi-structured interviews with 18 ACOs to understand their processes and strategies for caring for patients with complex health needs. In each ACO, we first conducted an interview with ACO leaders such as the director, chief medical officer, or other executive-level individual. All interviewed ACOs were invited to complete a second round of interviews with care managers, directors of care management programs, practice leaders, or others suggested by ACO executives. Eleven ACOs agreed to participate in follow-up interviews with frontline staff, and we conducted an additional 21 interviews. The second round of interviews aimed to identify individuals with on-the-ground experience of the ACO's approaches for caring for patients with complex health needs. The online appendix provides more detail on the characteristics of interviewed ACOs.

Interviews were conducted via telephone between February and June 2018. All interviews were recorded and transcribed and then analyzed using QRS NVivo. ${ }^{28}$ ACOs were selected from respondents to the National Survey of ACOs and limited to those with a Medicare Shared Savings Program (MSSP) contract that achieved shared savings in at least 1 year. $^{29}$ We used an iterative outreach process to achieve diversity in terms of geography, composition, ownership, and payer. Of the interviewed ACOs, 13 had at least one additional ACO contract with a commercial or Medicaid payer. Semistructured interviews lasted approximately $1 \mathrm{~h}$ and included information on the ACO structure, leadership, governance, engagement with primary care practices, and approaches to caring for complex patients.

We identified 11 ACOs that used care plans for patients with complex health needs. We defined a care plan as a written document created by a member of the patient's care team and developed based on interaction with the patient (i.e., not solely data driven). To be included in our analyses, care plans must have included information on the patient's medical history, current clinical needs, and future management of the patient. ${ }^{9}, 15,17$

Our analytic approach was collaborative and iterative. ${ }^{30}$ All transcripts were first coded by a research assistant and then coded unblinded by the first author, any coding discrepancies were discussed. We developed a detailed memo of results based on initial coding that identified themes and findings across ACOs, with examples to support each theme. The memo was iteratively revised based on team discussion and on further review and coding of the data.

\section{RESULTS}

In the 11 ACOs that used care plans, they were typically created as part of a broader care management program. Patients under care management included those (1) with frequent hospital-based utilization such as inpatient stays or emergency department visits, (2) with multiple chronic conditions, (3) with high costs, or (4) identified by algorithms or providers as high risk of costs or utilization. Care management staff-including medical assistants, health coaches, care 
managers, and care coordinators - were generally responsible for developing and maintaining care plans. While primary care and specialist physician providers may utilize or review care plans, they were not responsible for developing care plans at any of the interviewed ACOs.

\section{Core Functions and Scope of Care Plans}

The scope of care plans and the processes used to develop them varied along a continuum based on how ACOs described the core functions of care plans - ranging from care plans predominantly used as a tool to aid the care team to care plans as a tool for patient engagement (Fig. 1). Most ACOs used care plans as either a blend of condition and patient driven or as predominantly patient driven. Few used them as solely a tool to aid the care team in organizing and sharing patient information. These care plans typically functioned to provide a snapshot of the patient. For example, one ACO described their care plan as a "landing space" for providers, with a dashboard of important data points:

We've added customizations [to their health record], so you can quickly see the risk of readmission, other risk factors, the Gagne risk score, the care manager risk score, you can see a summary of their medications, you can see a summary of their encounters. It's somewhat of a landing place. (ACO executive)

At this end of the continuum, care plans typically relied on condition-based guidelines to develop patient goals and aimed to improve specific and measurable aspects of patient health. As part of their disease management program, one ACO used software developed from evidence-based clinical guidelines to automatically generate care plans and goals from the patient's history and current clinical markers. These care plans only addressed clinical needs and were minimally modified based on the patient's priorities; for example, goals were based on identified patient care gaps such as immunizations or upcoming lab tests.

Along the middle of the continuum, where care plans were designed to help the care team address clinical needs and as a tool to engage patients, care plans tended to be more comprehensive and required greater involvement of both staff and patients. These blended care plans typically involved non-clinical elements such as social needs and patient activation. One ACO included information about patients' medical conditions, preventive care needs, social needs (e.g., transportation or housing), substance use, and whether the patient wears glasses or hearing aids. Another ACO used standard patient assessment tools including patient activation measures. One ACO described:

We're able to go in at that time frame and do that comprehensive assessment, which then gives us the ability to see exactly what the problems are, whether it's a medical problem, whether it's a psychosocial problem, whether it's a behavioral health problem, and we can develop care plans that are really individually specific to the patient's needs, as well as interventions that we can employ to help those patients meet those goals of the care plans. (ACO care management staff)

At the other end of continuum, ACOs used care plans primarily as a patient coaching and engagement tool. While these ACOs included clinical aspects in their care plans, the motivation and approach were driven by patient coaching. One ACO created "shared action plans" after patients had a 2-h visit with care coordinators that focused on developing patient-centric goals such as walking to the mailbox or going on a vacation. Another ACO described their motivation for developing care plans:

Our care coordinators help patients set their own personal goals that they want to achieve as part of, not only what the doctor has indicated the goals they need to meet, but what are their own personal goals that they wanna reach? And ensuring that we're addressing their psychosocial as well as their clinical needs. We've seen that addressing their social determinants of health. Sitting down with them and figuring out, what are the barriers to care, what's causing them to visit the emergency room or not come in for a visit or reasons why

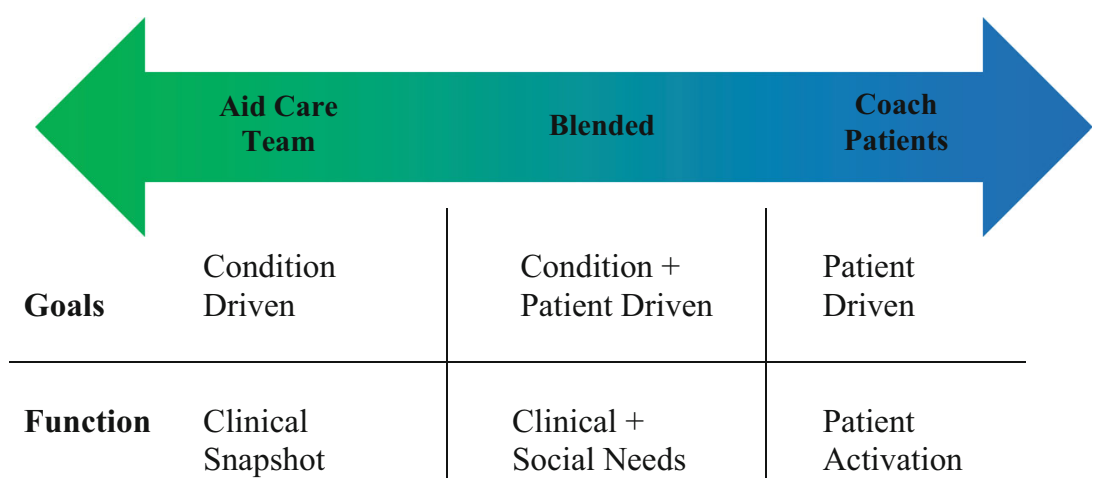

Figure 1 Scope and function of care plans used by ACOs. 
they don't pick up their medications, trying to identify those underlying issues. (ACO management)

\section{Care Plan Elements}

Patient History. Patient histories ranged in their comprehensiveness and included elements such as immunization records, lab and test results, utilization, past procedures, or social histories. ACOs prioritized and included patient information that they thought would be most useful in delivering care, as one ACO noted "whatever might be pertinent to that particular patient's health situation" (ACO executive). For example, one ACO initially focused primarily on the patient's social history, such as employment, living situation, and family status, with less of an emphasis on clinical markers.

Current Clinical Needs. Organizing and documenting current medical needs into a centralized location was often the predominant focus of care plans. Most ACOs included information about current medications - some were focused on listing medications, others regularly reviewed medication lists with patients, and some actively reconciled medications. In addition to general history, care plans highlighted information about recent healthcare utilization, especially costly hospital-based care to better understand patient needs. As one ACO explained:

One of the categories was making sure that the longitudinal plan of care served up ED visits and hospitalizations.... That [utilization] would be really relevant for a care team member who's interested in what's happening to the patient right now. (ACO executive)

Many ACOs included specialist care in the patient's care plan. In most cases, ACOs simply asked the patient for information to document their specialist providers as well as any upcoming visits. Other ACOs, such as those integrated with a larger system, had greater coordination with specialist care. For example, one ACO indicated patient care plans were fully accessible and integrated with both primary care and specialist care within their healthcare system.

Patient Goals. Care plans often included patient clinical goals that were aligned with specific conditions or patient needs such as controlling clinical markers. A couple ACOs described robust algorithms that used the patient's current health markers to generate clinical goals such as identifying specific targets to more optimally control blood pressure or blood sugar.

Seven ACOs referenced patient-developed goals as part of the care plan. One ACO explained:

Yeah, we let the patient talk freely about maybe a goal they want to set for themselves and we couple this with the understanding of where their engagement level is because if the patient PAM [patient activation] score is a level one, they're very disconnected from their health needs and they're not engaged so it might be learning what they are engaged with, or maybe they're worried about something, or one gentleman all he wanted to do was get to his granddaughter's first birthday and he was estranged from his daughter and there was a lot of dynamics there. But if you begin working on those and breaking down the barriers there you can build up a confidence level and a trust level with the care coordinator and pretty soon your interjecting, oh but if you try this [quit] line you could maybe not need your oxygen as often and not smoke. (ACO executive)

ACOs defined what was considered a patient goal differently. For some ACOs, patient developed goals meant that the patient prioritized which clinical goal to address. For other ACOs, goals could be completely patient generated and not necessarily clinical in nature. For example:

...pieces of their care plan that are specific to what might be important to them. We've had people say, 'It's important to me that I stay in my home,' so we might work with and recommend an in-home safety evaluation.... (ACO management)

\section{Access and Use of Plans by the Care Team}

ACOs offered little information on if and how care team members accessed patient care plans. Physicians often had limited engagement in care management programs at ACOs-which care plans were typically a part of - because care management programs were often centralized at the ACO level with most activities occurring independent of primary care (e.g., care managers directly calling patients). Interviewees did not see this as a challenge or limitation to care plans, but rather as a way to minimize burden on clinical care teams. Care plans were viewed and used as a valuable tool for care management staff as they operated in parallel with the physicians and other care team members.

Only two ACOs explicitly reported that patient care plans were accessed by physician providers: one of those ACOs actively included physicians in the development and implementation of standardized care plans. In this case, plans were first created by a care manager and then embedded in the electronic health record so that anyone accessing a patient's record first saw the care plan. Care plans were automatically updated when the patient's record was modified. Other ACOs embedded care plans in their care management platform. While physicians and other care team members could access those platforms, interviewees were uncertain if physicians actually looked at the care plans. One ACO specifically reported that they no longer gave access to all primary care physicians due to a lack of care management software interoperability with practices' electronic health records. 


\section{DISCUSSION}

Personalized, comprehensive patient care plans are promoted as a key tool to ensuring high-quality, advanced primary care is delivered by helping care teams manage patients with complex clinical or social needs through effectively addressing care needs across different settings and emphasizing patient goals and preferences. ${ }^{5,9-11,15-18,31-33}$ Most interviewed ACOs developed care plans as part of broader care management programs, and patient care plans were most commonly maintained and used by care managers. While all ACOs included the same core elements of care plans - the patient's history, current clinical needs, and future management goals - the depth and comprehensiveness of these elements varied. Some ACOs used care plans to predominately help the care team organize patient information while other ACOs also used care plans as a tool to engage patients. We observed three broad approaches to the way care plans were created and used: as a tool for providers, as a tool for patient engagement and coaching, and as a tool for both patients and providers.

Our findings suggest providers may struggle to implement care plans that are developed across care settings and are aligned with patient-driven goals. This may be due to separation of care management programming from clinical care coupled with care management-focused usage of care plans. Prior studies found that care plans can be modestly effective at improving patient outcomes and controlling costs when both providers and patients are engaged in the development and use of the plan. ${ }^{11}$ When care plans integrate the perspectives of both the patient and the care team, including primary and specialty care, they are more likely to optimally align clinical and patient goals. ${ }^{6,16,17}$, $19,33,34$ Yet, even though many ACOs approached care plans as a collaborative process between patients and the care team, several approached care plans primarily as a tool to organize patient care for the providers. While care plans should certainly serve as a centralized location to record patient care, the most successful plans also catalyze patient engagement. ${ }^{17}$ There is wide agreement among research findings and patient advocates that outcomes are improved when patients are actively engaged in the decision-making and planning around their care, but this was not consistently happening among the ACOs we interviewed. ${ }^{15,17,18,35}$

Team-based care is a widely used approach to manage the care of patients with complex clinical needs. ${ }^{35-38}$ It is not surprising that most of the ACOs we interviewed relied upon a range of care team members, such as care coordinators, medical assistants, and health coaches, to develop care plans. Non-clinicians extend the reach of primary care to ensure patients can receive more intensive and frequent care as needed. ${ }^{39,40}$ The optimal role and engagement between such care team members and clinicians in developing patient care plans is less certain. Only a couple of the ACOs we interviewed explicitly described if and how primary care clinicians regularly accessed patient care plans. At those ACOs, clinicians were centrally involved in patient care planning. In other interviewed ACOs, they noted primary care providers might or could have access to care plans, but did not suggest that the access was utilized, indicating clinicians were not centrally engaged in developing, reviewing, or implementing patient care plans. The work of documenting and developing care plans may not be the most efficient use of a clinician's time, yet clinician access to this information could help them deliver care that better incorporates patient's goals and coordinates across clinical settings. While it is uncertain how clinicians should be optimally engaged in such patient management activities, many suggest that high functioning care teams should regularly communicate with one another to ensure patient care is effectively coordinated. ${ }^{16,36,41}$

Our study highlights the challenges of coordinating care across several providers - care plans, and ACO goals more generally, aim to centralize and coordinate care across various settings and specialties. ${ }^{22}$ Patients with complex health needs may have several specialist providers in addition to their primary care provider-Medicare beneficiaries with two or fewer chronic conditions typically visit three clinicians in a year compared with eleven for those with seven or more chronic conditions ${ }^{2}$ - given challenges associated with care silos, lack of interoperability, and communication among providers, it is not surprising ACOs struggled to document specialist care within care plans. ${ }^{3,33}$ Most ACOs had to rely on patients to tell them about upcoming or recent specialist appointments. Only two ACOs, both part of highly centralized healthcare delivery systems, which are only half of ACOs in $2018,{ }^{42}$ implemented system-wide initiatives to fully integrate specialist and primary care clinicians into care plans. Even ACOs which include hospitals in their contract may struggle to coordinate care across settings, and they report similar care delivery capabilities as ACOs without a hospital. ${ }^{39}$ Given these obstacles, providers participating in ACOs and advanced primary care models, such as CPC+ and Primary Care First, may struggle to realize the potential of care plans without greater support from policymakers and others to address integration and communication challenges. ${ }^{12-14,} 32$

Care plans can serve as the centralized place for comprehensive clinical and social information on a given patient for the broader care team to access, review, and update as needed. ${ }^{5,}$,11, 15-18, 21, 31, 43 A group of key stakeholders recently developed a set of guidelines for care plans - they conceptualized care plans as proactively addressing patients' total health needs such that the plan becomes the cornerstone tool for identifying and developing ways to resolve patients' total health needs. ${ }^{15}$ Our findings on the role of the patient in developing the care plan, the accessibility of care plans by clinicians, and the challenges of coordinating across care settings raise questions on the centrality of patient care plans within ACO primary care today, where in theory practices should be most motivated to engage in these activities. ${ }^{44}$ Among ACOs we interviewed, care plans, even at their most comprehensive form, were still fragmented such that key pieces were either inaccessible or missing. 
Care plans which do not actively engage patients in the development of goals cannot proactively address patient needs, and plans that are not used by the entire care team, such as clinicians, cannot serve as the central basis for all patient care. Few, if any, of the ACOs we interviewed used care plans as policymakers, scholars, and others envisioned. To successfully evaluate the impact of care plans and establish best practices, researchers need to consider how healthcare organizations operationalize care plans in further studies.

Our study has limitations-first, as a qualitative study, our results should not be generalized to all ACOs or providers. These findings can provide insights into the approaches some ACOs use to implement personalized care plans and can be used to develop hypotheses about the value of care planning within ACOs. Some of our data are from the perspective of executives and managers at ACOs with less information from frontline clinicians which could impact our understanding of how much clinicians are involved in patient care plans. Finally, we do not have data on the effectiveness of these care plans because our study focused on identifying and describing the approaches used to implement care plans. ACOs indicated they believed patient care plans helped them provide better patient care.

Our study offers valuable insights for frontline clinicians and policymakers by identifying approaches ACOs use to implement care plans. Our study suggests that patient care plans may not always align with prescribed best practices: a tool to collaboratively develop centralized documentation and goals that integrate perspectives of primary care, specialist care, and the patient. Rather ACOs may be adapting care plans to create value for both their patients and their organization. Providers and payers should consider the optimal engagement of clinicians, other care team members, and patients in care planning. As clinicians experience increasingly complex patient populations, a changing healthcare environment, and policies aimed at controlling costs while increasing qualitythey need effective strategies to enhance care delivery.

Corresponding Author: Taressa K. Fraze, PhD; Department of Family and Community Medicine, University of California, San Francisco, San Francisco, CA, USA (e-mail: Taressa.Fraze@ucsf.edu).

Funding Information The authors are grateful for support from the Six Foundation Collaborative, whose members are the Commonwealth Fund (Grant No. 20171072), the Peterson Center on Healthcare (Grant No. 18011), the Robert Wood Johnson Foundation (Grant No. 74883), the SCAN Foundation (Grant No. 17-013), the John A. Hartford Foundation, and the Milbank Memorial Fund. This work was supported by the Agency for Healthcare Research and Quality (AHRQ)'s Comparative Health System Performance Initiative under Grant No. U19HSO24075, which studies how healthcare delivery systems promote evidence-based practices and patient-centered outcomes research in delivering care. Adam Briggs was a Harkness Fellow funded by the Commonwealth Fund. The views presented here are those of the authors and should not be attributed to The Commonwealth Fund or its directors, officers, or staff. Adam Briggs research at the University of Warwick is supported by the National
Institute for Health Research (NIHR) Applied Research Collaboration (ARC) West Midlands.

\section{Compliance with Ethical Standards:}

Conflict of Interest: The authors declare that they do not have a conflict of interest.

\section{REFERENCES}

1. Bynum JPW, Meara E, Chang C-H, Rhoads J, Bronner K. Our Parents, Ourselves: Health Care for an Aging Population. The Dartmouth Institute for Health Policy and Clinical Practice;2016.

2. Pham HH, Schrag D, O'Malley AS, Wu B, Bach PB. Care patterns in Medicare and their implications for pay for performance. N Engl J Med. 2007;356(suppl): 1130-1139.

3. Vogeli C, Shields AE, Lee TA, et al. Multiple chronic conditions: prevalence, health consequences, and implications for quality, care management, and costs. J Gen Intern Med. 2007;22 Suppl 3(Suppl 3):391-395.

4. Center for Medicare and Medicaid Services. Chronic Conditions among Medicare Beneficiaries: 2012 Edition. 2012.

5. Ganeshan S, Gheihman G, Palmor M, Choi S, Wohler D, Rotenstein LS. Shared care planning: Realizing the promise of team-based personcentered care. Healthc (Amst). 2018;6(2):110-111.

6. Grant RW, Adams AS, Bayliss EA, Heisler M. Establishing visit priorities for complex patients: A summary of the literature and conceptual model to guide innovative interventions. Healthcare (Amst). 2013;1(3-4): 117-122.

7. Rudin RS, Gidengil CA, Predmore Z, Schneider EC, Sorace J, Hornstein R. Identifying and Coordinating Care for Complex Patients: Findings from the Leading Edge of Analytics and Health Information Technology. (2162-8254 (Print)).

8. Schoen C, Osborn R, Squires D, Doty M, Pierson R, Applebaum S. New 2011 survey of patients with complex care needs in eleven countries finds that care is often poorly coordinated. (1544-5208 (Electronic)).

9. Coulter A, Entwistle V, Eccles A, Ryan S, Shepperd S, Perera R. Personalised care planning for adults with chronic or long-term health conditions. Cochrane Database Syst Rev. 2015;2015(3):CD010523. Published 2015 Mar 3. https://doi.org/10.1002/14651858.CD010523. pub2.

10. Lion KC, Mangione-Smith R, Britto MT. Individualized plans of care to improve outcomes among children and adults with chronic illness: a systematic review. Care Manag J. 2014;15(1):11-25.

11. Mercer T, Bae J, Kipnes J, Velazquez M, Thomas S, Setji N. The highest utilizers of care: individualized care plans to coordinate care, improve healthcare service utilization, and reduce costs at an academic tertiary care center. J Hosp Med. 2015; 10(7):419-424.

12. Department of Health and Human Services. Connected Care Toolkit. 2018; https://www.cms.gov/About-CMS/Agency-Information/OMH/ Downloads/connected-hcptoolkit.pdf. Accessed June 6, 2019.

13. Center for Medicare and Medicaid Innovation. Comprehensive primary care plus. 2016; https://innovation.cms.gov/Files/x/cpcplus-practiceslidepres.pdf. Accessed June 6ß, 2019.

14. Center for Medicare and Medicaid Innovation. Primary care first. 2019; https://innovation.cms.gov/Files/slides/pcf-info-webinar-series-slides. pdf. Accessed June 6, 2019.

15. Baker A, Cronin K, Conway PH, Desalvo KB, Rajkumar R, Press MJ. Making the Comprehensive Shared Care Plan a Reality. NEJM Catalyst. 2016; https://catalyst.nejm.org/making-the-comprehensive-sharedcare-plan-a-reality/. Accessed 2019, May 16.

16. Burt J, Rick J, Blakeman T, Protheroe J, Roland M, Bower P. Care plans and care planning in long-term conditions: a conceptual model. Prim Health Care Res Dev. 2014;15(4):342-354.

17. Edwards ST, Dorr DA, Landon BE. Can Personalized Care Planning Improve Primary Care? JAMA. 2017;318(1):25-26.

18. Lhussier M, Eaton S, Forster N, Thomas M, Roberts S, Carr SM. Care planning for long-term conditions - a concept mapping. Health Expect. 2015; 18(5):605-624.

19. Agency for Healthcare Research and Quality (AHRQ). Integrating Behavioral Health in Primary Care Playbook: develop a shared care plan. https://integrationacademy.ahrq.gov/products/playbook/developshared-care-plan. Accessed October 11, 2018. 
20. Lewis VA, Colla CH, Schoenherr KE, Shortell SM, Fisher ES Innovation in the Safety Net: Integrating community health centers through accountable care. J Gen Intern Med. 2014;29(11):1484-1490.

21. Reuben DB, Tinetti ME. Goal-oriented patient care-an alternative health outcomes paradigm. N Engl J Med. 2012;366(9):777-779.

22. Lewis VA, Schoenherr K, Fraze T, Cunningham A. Clinical coordination in accountable care organizations: A qualitative study. Health Care Manage Rev. 2018(1550-5030 (Electronic)).

23. Colla CH, Lewis VA, Kao L-S, O'Malley AJ, Chang C-H, Fisher ES. Association Between Medicare Accountable Care Organization Implementation and Spending Among Clinically Vulnerable Beneficiaries. JAMA Intern Med. 2016;176(8):1167-1175.

24. Wammes JJG, van der Wees PJ, Tanke MAC, Westert GP, Jeurissen PPT. Systematic review of high-cost patients' characteristics and healthcare utilisation. BMJ Open. 2018;8(9):e023113-e023113.

25. Tumlinson A, Burke M, Alkema G. The CHRONIC Care Act of 2018: Advancing Care for Adults with Complex Needs. The SCAN Foundation March, 20182018.

26. Center for Medicare and Medicaid Services. Connected Care: The Chronic Care Management Resource. 2020ß; https://www.cms.gov/About-CMS/ Agency-Information/OMH/equity-initiatives/chronic-care-management. Accessed February, 13, 2020.

27. Centers for Medicare and Medicaid Services. Innovation Models. https:// innovation.cms.gov/initiatives/\#views=models. Accessed February, 13, 2020

28. NVivo for Mac [computer program]. 2014.

29. Colla CH, Lewis VA, Shortell SM, Fisher ES. First National Survey of ACOs finds that physicians are playing strong leadership and ownership roles. health aff (Millwood). 2014;33(6):964-971.

30. Miles MB, Huberman AM, J S. Qualitative data analysis: A sourcebook of new methods. Thousand Oaks: Sage; 2014.

31. Reeves D, Hann M, Rick J, et al. Care plans and care planning in the management of long-term conditions in the UK: a controlled prospective cohort study. Br J Gen Pract. 2014;64(626):e568-e575.

32. Sessums LL, McHugh SJ, Rajkumar R. Medicare's Vision for Advanced Primary Care: New Directions for Care Delivery and Payment. JAMA 2016;315(24):2665-2666.

33. Tinetti ME, Esterson J, Ferris R, Posner P, Blaum CS. Patient Priority-Directed Decision Making and Care for Older Adults with
Multiple Chronic Conditions. Clin Geriatr Med. 2016;32(2):261275.

34. Tinetti ME, Naik AD, Dodson JA. Moving from disease-centered to patient goals-directed care for patients with multiple chronic conditions: Patient value-based care. JAMA Cardiol. 2016;1(1):9-10.

35. Council LS, Geffken D, Valeras AB, Orzano AJ, Rechisky A, Anderson S. A Medical Home: Changing the Way Patients and Teams Relate Through Patient-Centered Care Plans. Fam Syst Health. 2012;30(3): 190-198.

36. Leach B, Morgan P, Strand de Oliveira J, Hull S, Ostbye T, Everett C. Primary care multidisciplinary teams in practice: a qualitative study. BMC Fam Pract. 2017;18(1):115. Published 2017 Dec 29. https://doi. org/10.1186/s12875-017-0701-6.

37. Lemueux-Charles L, McGuire WL. What Do We Know about Health Care Team Effectiveness? A Review of the Literature. Med Care Res Rev. 2006;63(3):263-300.

38. Muntinga ME, Van Leeuwen KM, Schellevis FG, Nijpels G, Jansen APD. From concept to content: assessing the implementation fidelity of a chronic care model for frail, older people who live at home. BMC Health Serv Res. 2015;15:18.

39. Gorbenko KO, Fraze T, Lewis VA. Redesigning Care Delivery with Patient Support Personnel: Learning from Accountable Care Organizations. Int J Care Coord. 2016;19(3-4):73-83.

40. Lewis VA, Tierney KI, Fraze T, Murray GF. Care Transformation Strategies and Approaches of Accountable Care Organizations. Med Care Res Rev. 2019;76(3):291-314. https://doi.org/10.1177/ 1077558717737841

41. Hudon C, Chouinard MC, Diadiou F, Lambert M, Bouliane D. Case Management in Primary Care for Frequent Users of Health Care Services With Chronic Diseases: A Qualitative Study of Patient and Family Experience. Ann Fam Med. 2015;13:523-538.

42. Peck KA, Usadi B, Mainor AJ, Fisher ES, Colla CH. ACO Contracts With Downside Financial Risk Growing, But Still In The Minority. (15445208 (Electronic)).

43. McCarthy D, Ryan J, Klein S. Models of care for high-need, high-cost patients: an evidence synthesis. Commonw Fund. 2015;31:1-19.

44. McWilliams JM. Cost Containment and the Tale of Care Coordination. N Engl J Med. 2016;375(23):2218-2220.

Publisher's Note Springer Nature remains neutral with regard to jurisdictional claims in published maps and institutional affiliations. 